\title{
Reducing the Volume of Antibiotic Prescriptions: A Peer Group Intervention Among Physicians Serving a Community with Special Ethnic Characteristics
}

\author{
Rachel Wilf-Miron, MD, MPH; Naama Ron, RN, MSc; Shlomit Ishai, BA, Mgr; \\ Hana Chory, MD; Louis Abboud, MD; and Ronit Peled, MPH, PhD
}

\begin{abstract}
BACKGROUND: Antibiotics are a front-line weapon against many infectious diseases. However, antibiotic overuse is the key driver of drug resistance. Previously published studies have suggested benefits of using peer-topeer education, working with group leaders to build trust and maintain confidentiality within a quality initiative. We hypothesized that working with physicians as a peer group might be beneficial in influencing antibiotic prescribing patterns.
\end{abstract}

OBJECTIVE: To describe and evaluate a peer group model for an intervention to reduce the volume of antibiotic prescriptions among physicians with above average prescribing rates serving an Arab community in northern Israel.

METHODS: Primary care physicians in a defined geographic area who served Arab communities and had high antibiotic prescribing ratesdefined as above average number of antibiotic prescriptions per office visit compared with regional and organizational averages-were recruited for the intervention. All other physicians from the same region served as a comparison group. The intervention was administered during 2007 and was completed in early 2008. Four structured meetings scheduled 2 months apart, in which the group explored the issues related to antibiotic overuse, included the following topics: adherence to clinical guidelines; the special position physicians serving Arab communities hold and the influence it has on their practices; pressure due to consumer demands; and suggestions for possible strategies to face ethnic sensitivity, mainly because of the special ties the physicians have with their communities. T-tests for independent samples were used to perform between-group comparisons for each quarter and year of observation from 2006 through 2010, and t-tests for paired samples were used to compare pre-intervention with post-intervention antibiotic prescribing rates.

RESULTS: In the 2006 pre-intervention period, the antibiotic prescribing rates were 0.17 for the peer group ( $n=11$ physicians) and 0.15 for the comparison group ( $\mathrm{n}=72$ physicians, $P=0.279$ ). In 2008 following the intervention, rates were 0.12 and 0.14 , respectively $(P=0.396)$. In the paired t-test analysis, rates declined significantly from 2006 to 2008 in the intervention group $(P<0.001)$ but not in the comparison group $(P=0.138)$. Antibiotic prescribing rates remained similar in 2009 and 2010.

CONCLUSION: In the context of a community with special ethnic and cultural characteristics, an intervention relying on peer group techniques was associated with a modest reduction in the volume of antibiotic prescriptions.

J Manag Care Pharm. 2012;18(4):324-28

Copyright $\odot 2012$, Academy of Managed Care Pharmacy. All rights reserved.
A ntibiotics are a front-line weapon against many infectious diseases. However, antibiotic overuse is the key driver of drug resistance. Paradoxically, this adverse phenomenon has resulted from the combination of overuse, particularly for minor infections, and underuse because of failure to complete the treatment course., ${ }^{1,2}$ Yet, some general practitioners (GPs) do not regard prevention of antibiotic resistance as an important priority in their clinical practices. ${ }^{3}$ Others are concerned that not prescribing antibiotics may negatively affect clinician-patient relationships. ${ }^{4}$ It has been suggested that perceived patient demand has a significant, independent, and clinically relevant effect on antibiotic prescribing 5 and that patients often overestimate the benefits of antibiotics. ${ }^{6}$

Maccabi Healthcare Services (MHS) is the second largest health maintenance organization (HMO) in Israel. It provides community-based health services to 1.9 million members from diverse population sectors throughout the country. Services are provided through 5 regions and 150 branches (the local administrative unit) and are based primarily on self-employed physicians working in solo practices. The population in the northern region of the country, where the present study intervention was implemented, comprises 50\% of Israel's Arab citizens, who live in rural settlements and mixed cities such as Nazareth and are entitled to equal health care services under the National Health Insurance Law. MHS services to its Arab members in the region are provided through the Nazareth branch by self-employed physicians, most of them of Arab ethnicity. The Arab society in the region is characterized by low socioeconomic status, tight family and neighboring relationships, and close community relationships (mostly through families) with physicians. These characteristics may affect health care delivery.

In 2007, the MHS regional management observed that GPs serving the Arab population in the Nazareth area were, in general, prescribing antibiotics at higher rates per visit when compared with the rest of the northern region and MHS averages. This observation, coupled with the large variation in prescription rates among those physicians, prompted the study intervention.

While planning the intervention, our assumptions were as follows: (a) antibiotic use is a clinical issue and mostly under the physician's control; (b) Arab physicians, who hold a special social position in their communities, are under significant 
pressure to prescribe antibiotics due to close community ties and relationships; and (c) antibiotic use can be changed by clarifying and strengthening medical practice in conjunction with reducing consumer pressure. These assumptions, along with the findings of previously published research regarding the benefits of using peer-to-peer education in working with group leaders to build trust and maintain confidentiality within quality initiatives, ${ }^{7}$ led us to postulate that working with physicians as a peer group might be beneficial in influencing antibiotic prescribing patterns.

A peer group is defined as a group of equals; a social entity consisting of people who are similar in features such as age, education, social class, or some other variable. ${ }^{8}$ Physicians can be defined as a "peer group" when they work in similar conditions and care for patients of similar background in the context of the same health care system. This group can serve as a platform for quality improvement. ${ }^{9}$ The project objective was to use a peer group model for an intervention to reduce the volume of antibiotic prescriptions among physicians with above average prescribing rates serving an Arab community.

\section{Methods}

\section{Intervention}

In the 3 years preceding the intervention, MHS established an infrastructure to promote quality improvement. This system has been previously described ${ }^{10,11}$ and included the training of regional and branch multidisciplinary "quality teams" to identify gaps in performance and conduct interventions to improve care. Each regional team was guided by a quality facilitator (a coach) who was experienced in quality improvement methods. The intervention was conducted by MHS in the Nazareth District (northern Israel) and was launched in 2007. The first 3 months were dedicated to preparing the intervention and recruiting the participants. Four structured meetings of 4 hours each and 2 months apart were scheduled and conducted until mid-2008.

Recruitment. Thirteen GPs serving Arab patients (11 male Arab physicians and 2 female non-Arab physicians) were invited by the branch medical director to participate in the group meetings in order to improve antibiotic prescribing. Eleven (10 males and 1 female) accepted the invitation. Inclusion criteria included the following: (a) demonstration of higher rates of antibiotic prescribing compared with regional and organizational averages, (b) having medium to large practice sizes (500-3,000 registered beneficiaries), and (c) willingness to attend the group meetings for the purpose of care improvement. In 2008 and 2010, these physicians had 44,375 and 47,500 MHS patient visits, respectively. Patients, both pediatric and adult beneficiaries, were of all ages.

Ethics. The intervention was approved by the MHS Department of Quality Management in Healthcare and did not require any further approval. The peer group consisted of physicians who agreed to participate, and no consent forms were required.

Group Work Structure. The group was organized under the leadership of the branch medical director who, as a practicing GP, agreed to lead the local quality improvement initiative The entire process was guided by a quality facilitator, who was a registered nurse with expertise in quality improvement concepts and methodologies. The physicians who agreed to participate were committed to work as a group for the purpose of improving their clinical performance as individuals and, eventually, as a group.

\section{Intervention Meeting Structure and Contents}

Each meeting started with a presentation of antibiotic prescription data, including the group's average and variations among individual physicians, followed by an analysis of enablers and barriers to change. In order to encourage open and honest discussion, individual data were presented by code number instead of a name to ensure confidentiality and anonymity; participants knew only their own code numbers, enabling them to compare themselves with colleagues without being identified. Later, an expert in infectious diseases was invited to present a talk on issues regarding good practice and antibiotic prescribing guidelines.

During the meetings, the following topics were discussed: difficulties in implementing antibiotic prescribing guidelines; participants' dilemmas regarding patient-physician relationships in a competitive market; the community's cultural context, where a "good" physician is one who prescribes antibiotics "on demand," differences in antibiotic prescribing due to high rates of infections resulting from external injuries that are common in the region; ${ }^{12}$ and frequent antibiotic prescriptions by local dentists.

Opportunities were also provided to share on issues such as dealing with patients' needs and demands in the context of a short clinical encounter; addressing the special role a physician plays in Arabic society according to social codes related to the culture and tradition of respect and honor; and physicians' commitment to their peers and the community. With time, the physicians were molded into a tight-knit peer group that could determine the program's targets. As a group, they began using the local media to explain the possible harms of antibiotic overuse. They also created a telephone network between themselves and the MHS regional pharmacist for use as a tool to manage their own practices. For example, the following took place at a meeting:

- The medical director presented the latest data analysis revealing group average and individual peer antibiotic prescribing rates.

- The participants discussed how recommendations from the previous meeting were implemented and shared their experiences regarding these recommendations. 
- The participants discussed antibiotic prescribing issues and shared their experiences in handling difficulties arising when refusing patient demands for treatment with antibiotics.

- The group discussed whether to prescribe antibiotics before receiving throat culture results, while taking the possibility of an additional office visit into account.

- The participants discussed their frustration when detailed explanations on antibiotic prescriptions are needed and the patient encounter is short.

- The group discussed the role physicians play in a conservative community with strong family ties and the social and personal status each possesses in his/her own community.

- The group formulated a set of recommendations for how to achieve an appropriate antibiotic prescribing rate without damaging relationships with patients (e.g., maintaining telephone contact with the patient after a clinic visit).

- The group formulated an agreement in which each member committed not to accept "wandering patients" who move from one physician to another.

\section{Intervention Evaluation}

Comparison Group. All other physicians from the same region $(n=72)$ served as a comparison group. Both groups (intervention and comparison) were composed of primary care physicians who practiced in similar conditions, such as type of practice (solo), location (mixed urban-rural setting), reimbursement method (self-employed practitioners), and system characteristics (fully computerized medical records).

Sample Size Calculations. In a priori power calculations, we assumed detection of a difference between means (standard deviations) of $0.15(0.05)$ and $0.13(0.06)$ in the intervention and comparison groups, respectively. Assuming alpha of 0.05, the number of cases needed for 50\% power (type 2 error rate of $50 \%)$ was 41 in each group. However, we succeeded in recruiting only 11 physicians for the intervention group.

\section{Data Analysis}

MHS has fully computerized records, including records of physician visits and pharmaceutical dispensing. Data were gathered from the regional registries using each physician's identification code. Monthly rates of antibiotic prescriptions (number of prescriptions per visit) were calculated for each primary care physician working in the Nazareth region (intervention group: 11 physicians participating in the peer group; comparison group: 72 nonparticipating physicians). We divided each year into 4 quarters and analyzed the 5-year period from 2006 through 2010. The pre-intervention period was considered to be 2006; 2007 and the first 2 quarters of 2008 were considered the intervention period (preparation and peer group meetings); and the last 2 quarters of 2008, the 4 quarters of 2009, and the

\section{TABLE 1 Mean [SD] Antibiotic Prescribing Rates $^{\mathrm{a}}$ for Intervention and Nonintervention Groups}

\begin{tabular}{l|c|c|c}
\hline \multicolumn{1}{c|}{ Year } & $\begin{array}{c}\text { Intervention } \\
\mathbf{n}=\mathbf{1 1}\end{array}$ & $\begin{array}{c}\text { Comparison } \\
\mathbf{n}=\mathbf{7 2}\end{array}$ & $\boldsymbol{P}^{\text {Value }}$ \\
\hline 2006 & $0.17[0.05]$ & $0.15[0.06]$ & 0.279 \\
\hline 2007 & $0.14[0.04]$ & $0.13[0.05]$ & 0.574 \\
\hline 2008 & $0.12[0.03]$ & $0.14[0.05]$ & 0.396 \\
\hline 2009 & $0.13[0.05]$ & $0.15[0.06]$ & 0.448 \\
\hline Change from 2006 to 2008 & $0.12[0.06]$ & $0.12[0.04]$ & 0.602 \\
\hline P value (2006-2008)c & 0.05 & 0.01 & \\
\hline
\end{tabular}

aThe antibiotic prescribing rate was defined as the number of antibiotic prescriptions divided by the total number of physician visits.

${ }^{b}$ T-test for independent samples.

'T-test for related samples.

SD = standard deviation.

first 3 quarters of 2010 were considered the post-intervention period. T-tests for independent samples were used to compare antibiotic prescribing rates between the groups for each time period. Paired t-tests were used to compare pre-intervention and post-intervention prescribing rates within each of the 2 study groups. Analyses were performed using SPSS, version 17 (IBM SPSS, Armonk, NY) and an a priori alpha of 0.05 .

\section{Results}

In the 2006 pre-intervention period, the antibiotic prescribing rates were 0.17 for the peer group ( $n=11$ physicians) and 0.15 for the comparison group ( $\mathrm{n}=72$ physicians, $P=0.279$; Table 1 and Figure 1). In 2007, rates were 0.14 and 0.13 , respectively $(P=0.574)$. In the paired t-test analysis, rates declined significantly from 2006 to 2008 in the intervention group $(P<0.001)$ but not in the comparison group $(P=0.138)$.

\section{Discussion}

Research on quality improvement theories has demonstrated that most individuals pursue high-quality work as a personal value; it is only necessary to supply adequate data and training to achieve high-quality results. This assertion may be even more true in medicine, which tends to attract those with a personal commitment to serve. ${ }^{13}$ The data analysis in the present study revealed an association between the implementation of the peer group methodology and a modest reduction in the volume of antibiotic prescribing rates among the participating physicians. Application of this methodology may potentially help address issues that result in overuse of antibiotics and other drugs in primary care.

Several factors contributed to the intervention's success. First, implementation of this innovative practice improvement method required organizational commitment by senior management, regional and branch leaders, and involvement 


\section{FIGURE 1 Antibiotic Prescribing Rates ${ }^{\mathrm{a}}$ for Intervention and Comparison Groups by Calendar Ouarter}

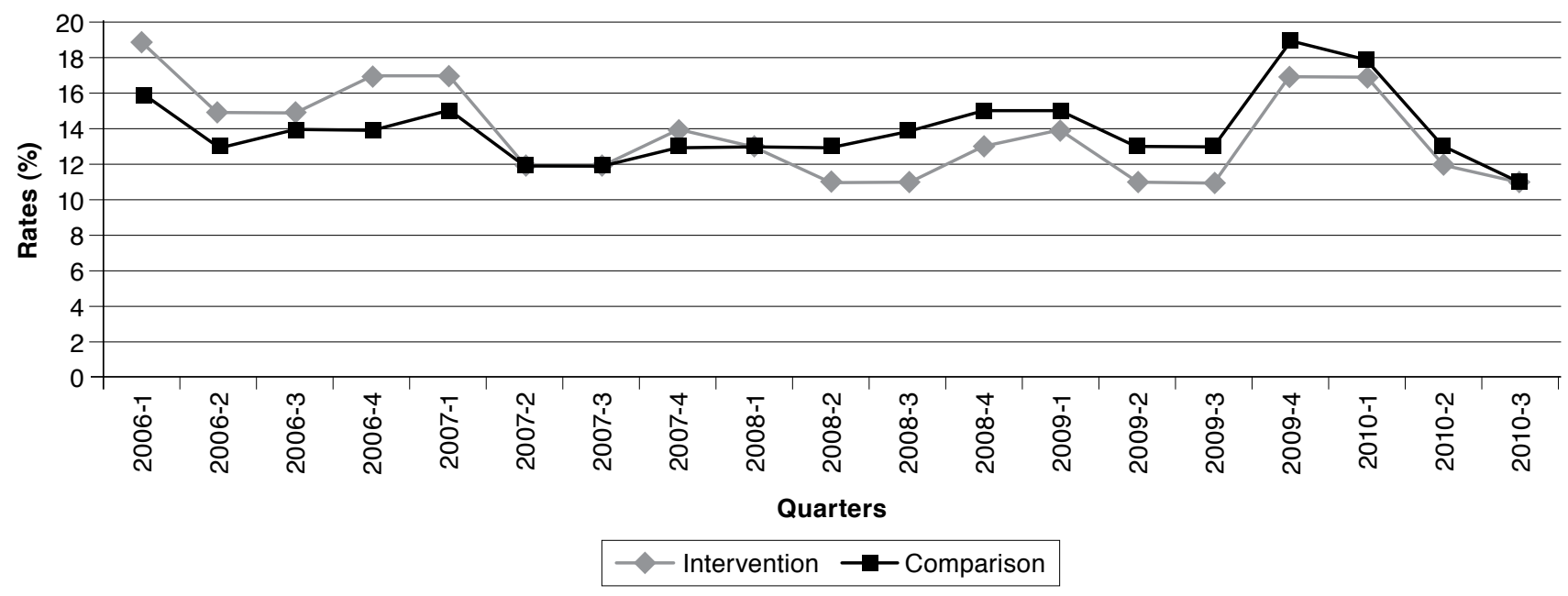

aThe antibiotic prescribing rate was defined as the number of antibiotic prescriptions divided by the total number of physician visits.

of a multidisciplinary team (GP, quality facilitator, pharmacist, and infectious disease specialist). Second, creation of an atmosphere of trust and collaboration was essential because all participating physicians were self-employed and reimbursed according to practice size (per capita reimbursement); thus, overt or covert competition for patients in the same area could have created a potential threat to cooperative group work. This atmosphere of trust was promoted in 2 major ways. The first was the decision to focus on clinical processes in the meetings rather than on placing blame. The second was the anonymous displaying of performance levels, which helped to create the nonjudgmental environment required for open and honest discussions.

In this atmosphere, the participants were ready to disclose difficulties and frustration, such as "being left alone to improve performance while not receiving enough support from the 'system," or the need for guideline modification in order to adjust recommended therapies to meet the clinical needs of patients. When trust was created among physicians as a group and especially between the group and representatives of the "system," physicians were willing to go forward with change. From that point on, they developed tools to help each other and to better communicate with their patients.

Another crucial element was the support for the local initiative expressed by MHS central quality management. They provided the local organizers with professional supervision and guidance throughout using a "quality facilitator" dedicated to the project. This facilitation strengthened the ties between the local physicians and MHS management to promote change. Finally, the peers' commitment as individuals and as a group to make necessary changes was also an important contributor to success.

Spooner et al. (2001) investigated the factors that motivated British GPs to take part in a quality improvement scheme. ${ }^{13}$ Contributing factors included a "desire to improve patient care," retention of "professional autonomy" and "professional pride," and peer pressure. They also suggested that when "managerial vision is aligned to professional values, and combined with a range of interventions... including financial incentives," professional practice can be influenced. Most of these factors, excluding financial incentives, played a role in our intervention, introduced to achieve physician commitment to improved quality of care.

In the present study setting, cultural factors also played a part. Arab physicians and other health care professionals who serve Arab communities are unique in the special role they play and the social position they enjoy in their communities. They frequently have family and neighborhood ties with their patients. This situation has led physicians to adjust their practice to patient demands, sometimes at the expense of disregarding professional treatment guidelines. Creation of a peer group provided physicians with the support that they needed to practice in a way that was more consistent with their professional evidence-based values.

James (2004) describes how he created an environment of professional accountability at Intermountain Healthcare (Salt Lake City, UT) in which groups of physicians and other professionals who manage similar patients in similar settings discuss best patient care practices. ${ }^{14}$ Within this setting, professionals had access to the medical literature, expert opinions, and 
credible data showing their own performance and outcomes. James states that this approach may be treated as "a redefinition of traditional peer review." 14 Our limited intervention supports his claim.

\section{Limitations}

First, the intervention we present was initiated on a small scale. However, the literature indicates that even limited quality improvement initiatives adopted by a small group can be disseminated and contribute to the creation of an environment supporting larger improvement effects. ${ }^{14}$ We therefore view the qualitative results obtained from this intervention as the starting point for further research and policy formation. Second, the intervention included physicians who, by agreeing to come forward and participate, were already committed to reducing their own antibiotic prescribing. Third, because the intervention group was selected on the basis of above average antibiotic prescribing rates, the study outcomes may be partially attributable to regression to the mean.

\section{Conclusion}

A peer group technique encouraged open dialogue within a group of independent primary care physicians and between the group and the health care system. This activity was associated with a modest reduction in antibiotic prescribing rates.

\section{Authors}

RACHEL WILF-MIRON, MD, MPH, is Director, Quality Management in Health Care; NAAMA RON, RN, MSc, is Quality Facilitator; SHLOMIT ISHAI, BA, Mgr, is Regional Pharmacist; HANA CHORY, MD, is Medical Manager, Northern Region; and LOUIS ABBOUD, MD, is Quality Manager and Medical Manager, Northern Region, Maccabi Healthcare Services, Nazareth, Israel. RONIT PELED, MPH, PhD, is Senior Lecturer and Researcher, Ben Gurion University of the Negev, Beer Sheva, Israel.

AUTHOR CORRESPONDENCE: Rachel Wilf-Miron, MD, MPH, Director, Quality Management in Health Care, Maccabi Healthcare Services, Tel-Aviv, 68125, Israel. Tel.: 972.3.5143753; Fax:

972.3.5143554; E-mail: rachel_m@mac.org.il.

\section{DISCLOSURES}

The authors report no financial or other conflicts of interest pertaining to the subjects or products discussed in this article.

Concept and design were performed primarily by Wilf-Miron with the assistance of Ron, Chory, and Abboud. The data were collected by Ishai and interpreted primarily by Peled and Wilf-Miron with the assistance of the other authors. The manuscript was written primarily by Wilf-Miron with the assistance of Peled and was revised by primarily by Wilf-Miron with the assistance of the other authors.

\section{ACKNOWLEDGEMENT}

The authors would like to acknowledge Ms. Orna Shem-Tov, MA, a strategic consultant to the Department of Quality Management in Healthcare, for her contribution to the success of the peer group intervention.

\section{REFERENCES}

1. Seppälä H, Klaukka T, Vuopio-Varkila J, et al. The effect of changes in the consumption of macrolide antibiotics on erythromycin resistance in group A streptococci in Finland. Finnish Study Group for Antimicrobial Resistance. N Engl J Med. 1997;337(7):441-46. Available at: http://www.nejm.org/doi/ full/10.1056/NEJM199708143370701. Accessed March 29, 2012

2. World Health Organization. WHO global strategy for containment of antimicrobial resistance. 2001. Available at: http://www.who.int/drugresistance/ WHO_Global_Strategy_English.pdf. Accessed March 29, 2012.

3. Simpson SA, Wood F, Butler CC. General practitioners' perceptions of antimicrobial resistance: a qualitative study. J Antimicrob Chemother. 2007;59(2):292-96. Available at: http://jac.oxfordjournals.org/content/ 59/2/292.long. Accessed March 29, 2012.

4. Butler CC, Rollnick S, Pill R, Maggs-Rapport F, Stott N. Understanding the culture of prescribing: qualitative study of general practitioners' and patients' perceptions of antibiotics for sore throats. BMJ. 1998;317(7159): 637-42. Available at: http://www.ncbi.nlm.nih.gov/pmc/articles/PMC28658/ ?tool=pubmed. Accessed March 29, 2012.

5. Coenen S, Michiels B, Renard D, Denekens J, Van Royen P. Antibiotic prescribing for acute cough: the effect of perceived patient demand. Br J Gen Pract. 2006;56(524):183-90. Available at: http://www.ncbi.nlm.nih.gov/pmc/ articles/PMC1828261/?tool=pubmed. Accessed March 29, 2012.

6. Cals JW, Butler CC, Hopstaken RM, Hood K, Dinant GJ. Effect of point of care testing for $C$ reactive protein and training in communication skills on antibiotic use in lower respiratory tract infections: cluster randomised trial. BMJ. 2009;338:bl374. Available at: http://www.ncbi.nlm.nih.gov/pmc/ articles/PMC2677640/?tool=pubmed. Accessed March 29, 2012

7. Gosfield AG, Reinertsen JL. Sharing the quality agenda with physicians. Trustee. 2007;60(9):12-14,16-17,1.

8. BusinessDictionary.com. Peer group definition. 2012. Available at: http:// www.businessdictionary.com/definition/peer-group.html. Accessed March 29, 2012

9. James BC. Quality management for health care delivery. The Hospital Research and Educational Trust of the American Hospital Association. 1989 Available at: http://intermountainhealthcare.org/qualityandresearch/institute/Documents/articles_qmmp.pdf. Accessed March 29, 2012.

10. Wilf-Miron R, Galai N, Gabali A, et al. Organizational efforts to improve quality while reducing healthcare disparities: the case of breast cancer screening among Arab women in Israel. Qual Saf Health Care. 2010;19(5):e36. Epub 2010 Jun 17. Available at: http://qualitysafety.bmj.com/ content/19/5/1.7.full.pdf+html. Accessed March 29, 2012.

11. Wilf-Miron R, Peled R, Yaari E, et al. Disparities in diabetes care: role of the patient's socio-demographic characteristics. BMC Public Health. 2010;10:729. Available at: http://www.ncbi.nlm.nih.gov/pmc/articles/ PMC3004835/?tool=pubmed. Accessed March 29, 2012.

12. Health conditions of the Arab population in Israel 2005. Available at: http://www.old.health.gov.il/Download/pages/arab2004_291109.pdf Accessed March 29, 2012.

13. Spooner A, Chapple A, Roland M. What makes British general practitioners take part in a quality improvement scheme? J Health Serv Res Policy. 2001;6(3):145-50

14. James BC. Three Methods to Manage Clinical Care. Course guidebook. Advanced Training Program (ATP) in health care delivery improvement. Salt Lake City, UT: Intermountain Healthcare; 2004. 